\title{
PERSPECTIVAS ECOFEMINISTAS Y ECOSOCIALISTAS SOBRE EL CAOS DEL CAMBIO CLIMÁTICO: AMENAZAS A LA MADRE TIERRA
}

\section{ECOFEMINIST AND ECOSOCIALIST PERSPECTIVES ON CLIMATE CHANGE CHAOS: THREATS TO MOTHER EARTH}

\author{
Ana Isla*
}

RESUMEN

El objetivo de este texto es articular el cambio climático, las causas de su origen y las posibilidades de superar esta crisis. El desarrollo de este documento se basa en las investigaciones de la autora y en el curso de Environmental Justice de la Brock University. Este curso presenta la perspectiva ecofeminista, la cual sostiene que el proceso de reproducción de la vida ha ocurrido por millones de años sin mayor alteración, sin embargo con el proceso de producción de mercancías que usa el combustible fósil, el monóxido de carbono $\left(\mathrm{C}_{2}\right)$ se acumula rápidamente en la atmósfera, creando la crisis del ecosistema. Para finalizar se presentan algunas perspectivas que proponen la Justicia Social como salida de la crisis ecosistémica.

PALABRAS CLAVE: ECOFEMINISMO * ECOSOCIALISMO * CAMBIO CLIMÁTICO * CRECIMIENTO ECONÓMICO * MONÓXIDO DE CARBONO

\section{ABSTRACT}

The objective of this article is to understand why climate change is occurring and how it can suddenly shift the state of the Earth's systems. The development of this document is based on the author's research and the Environmental Justice course of the Brock University. This course presents the ecofeminist perspective, which argues that the process of reproduction of life has occurred for millions of years without major alteration, but with the process of producing goods that use fossil fuels, carbon monoxide $\left(\mathrm{C}_{2}\right)$ accumulates rapidly in the atmosphere, which creates the ecosystem crisis. Finally, some perspectives that propose Social Justice to overcome the ecosystem crisis are presented.

KEYWORDS: ECOFEMINISM * ECOSOCIALISM * CLIMATE CHANGE * ECONOMIC GROWTH * DIOXIDE DE CARBONO 


\section{INTRODUCCIÓN}

En general, el cambio climático es una modificación en los patrones climáticos globales. Diversas causas de origen natural pueden hacer que el clima se modifique, como por ejemplo, la distancia de la Tierra al Sol, la cual aumenta o disminuye según su recorrido por la elipse; de la misma forma, los océanos o la Tierra pueden cambiar cuando un volcán entra en erupción. Sin embargo, la mayoría de los científicos están de acuerdo en que los humanos también pueden alterar el clima (Steffen, Crutzen y McNeill, 2007), porque la civilización es un motor de energía caliente, ya sea que use paneles solares o combustibles fósiles. Al respecto, actualmente existe un consenso científico acerca de que el cambio climático se debe al uso de combustibles fósiles: "Quemar estas cosas pone los gases en el aire. Los gases hacen que el aire se caliente. Estos gases pueden cambiar el clima de un lugar, pero también pueden cambiar el clima de la Tierra" (National Aeronautics and Space Administration, 14 de mayo de 2014). Sin embargo, a pesar de que la mayoría de los científicos coinciden en que los humanos pueden cambiar el clima, algunos no están de acuerdo en relación con qué o quién lo está causando, pero este debate no se desarrollará en este artículo. En este texto se limita a sostener que el calentamiento global es una de las fronteras planetarias para mantener la vida en la Tierra. Otras fronteras son: la acidificación oceánica, el agotamiento del ozono estratosférico, los ciclos de nitrógeno y fósforo, el uso global de agua dulce, el cambio en el uso de la tierra, la pérdida de biodiversidad, la carga atmosférica de aerosoles y la contaminación química.

La respuesta al cambio climático ha sido variada; las instituciones, a través de las Naciones Unidas, han articulado su respuesta en el documento Our common future (OCF) (WCED, 1987), en el cual se identificó tres agentes del calentamiento global: la pobreza, el desarrollo desigual y el crecimiento demográfico, todos consecuencia del crecimiento económico, porque la civilización que produce mercancías es un motor de energía caliente, tal como se lo define $y$ persigue actualmente. OcF ofrece en cambio un "crecimiento económico sostenible" o "capitalismo verde" mediante la extensión de los mercados en la naturaleza (como el ecoturismo o el crédito de carbono desde la foresta) como la solución. Desde que la onU adoptó este enfoque en la Cumbre de la Tierra de 1992, la "supervivencia del planeta" se ha convertido en la justificación para una nueva ola de intervenciones, por medio de las cuales las personas y la naturaleza se convierten en un dominio de la política (Sachs, 2010).

Asimismo, el Reporte del Panel sobre el Cambio Climático (Intergovernmental Panel on Climate Change, octubre, 2018) sostiene que el mundo necesita limitar la temperatura a 1.5 grados centígrados para evitar el devastador impacto, lo cual es sumamente conservador. Al nivel de los acuerdos políticos, el planeta está en curso de llegar a 2 o mas grados centígrados de calentamiento. El profesor Guy McPerson (31 de mayo de 2018) de la Universidad de Arizona, sostiene que el deshielo del Ártico, pronosticado para el año 2026, terminará con la producción de granos $y$, consecuentemente, con la civilización occidental. Un Océano Ártico sin hielo significa la perdida de hábitat para sobrevivir.

Nadie puede negar que el cambio climático ya está en marcha, cuyas consecuencias son impredecibles. Las transformaciones en los procesos físicos, químicos y biológicos de la Tierra son evidentes en todas partes, mientras las emisiones de gases de efecto invernadero han aumentado la concentración del ciclo del carbono en la atmósfera. En el pasado, la mitad de este carbono se almacenaba en los bosques, mientras que la otra mitad era eliminada por los océanos, pero con la industrialización creciente, la deforestación y el calentamiento de los mares, el oxígeno está en su punto más bajo. De esta forma, según la NASA y la Administración Nacional Oceánica y Atmosférica de los Estados Unidos (National Aeronautics and Space Administration, 18 de enero de 2017), "la temperatura superficial promedio del planeta ha subido aproximadamente 2.0 grados Fahrenheit (1.1 grados Celsius) desde finales del siglo XIx" (párr. 6). Por ejemplo, 2018, 2017 y 2016 fueron los años 
más cálidos registrados y rompieron récords establecidos en los años 2015 y 2014.

En enero de 2017, en el twitter del Parque Nacional Badlands se publicaron datos sobre el cambio climático a pesar de las órdenes dadas por el presidente Trump. Algunas de sus cifras son:

La concentración preindustrial de dióxido de carbono en la atmósfera era de 280 partes por millón (ppm). A diciembre de 2016, fue de $404.93 \mathrm{ppm}$.

Hoy, la cantidad de dióxido de carbono en la atmósfera es más alta que en cualquier momento en los últimos 650000 años.

La acidez del océano ha aumentado 30\% desde la Revolución Industrial.

Como resultado, la degradación ecológica es global y la Tierra se está volviendo cada vez más inhóspita y convirtiéndose en un escenario de eventos climáticos sin precedentes. La temperatura cambiante altera el equilibrio de las comunidades y degrada los ecosistemas. Por ejemplo, en Puno (Perú), entre julio y agosto de 2015, como resultado del descenso de las temperaturas a $-20{ }^{\circ} \mathrm{C}$, murieron 17 niños $y$ alrededor de 171000 alpacas por hipotermia, diarrea, neumonía e inanición. Según Sánchez (11 de julio de 2015), el Gobierno peruano se encuentra poco preparado para enfrentar grandes crisis, por lo que el apoyo fue tardío e inadecuado. Mientras tanto, en Alberta (Canadá), a causa de un invierno más seco combinado con una masa de aire inusualmente caliente $y$ seca proveniente del Norte de la región, la temperatura subió a $32,8{ }^{\circ} \mathrm{C}$ (Gobierno de Canadá, 06 de mayo de 2016). Como consecuencia, en mayo de 2016, hubo 49 incendios forestales activos, entre ellos, uno en Fort McMurray que se salió de control y cubrió 522892 hectáreas, incluyendo 2496 en Saskatchewan. Por lo cual, el Gobierno decretó la evacuación obligatoria de varias ciudades. La lucha contra el fuego en la provincia estuvo a cargo de 2794 bomberos $y$ personal de apoyo, 147 helicópteros, 16 buques cisterna y 233 piezas de equipo pesado (Alberta Government, 10 de junio de 2016). Mientras tanto, en el verano de 2017, tuvieron lugar cientos de incendios forestales en las provincias de British Columbia, Manitoba y Saskatchewan. Asimismo, en el verano de 2018, se registró altas temperaturas que llegaron entre $30{ }^{\circ} \mathrm{C} y$ $35{ }^{\circ} \mathrm{C}$, pero debido a la humedad, la sensación de calor fluctuó entre los $40{ }^{\circ} \mathrm{C}$ y $45^{\circ} \mathrm{C}$ por casi tres semanas consecutivas. A un ritmo creciente, incendios forestales cada más agresivos se expanden por Canadá y Estados Unidos (EE.UU.).

Además, en la superficie de los océanos están sucediendo con mayor frecuencia eventos destructivos debido al calentamiento. Según Davis (2017), los investigadores han documentado el dramático cambio en las concentraciones de oxígeno debido a las perturbaciones en el ciclo global del carbono. Cuando los océanos se calientan contienen menos gases disueltos, incluido el oxígeno, lo que afecta a los organismos marinos, especialmente a los mamíferos. El mayor volumen de oxígeno se perdió en el Pacífico debido a las detonaciones nucleares ocurridas entre los años 40 y 60 (Bertell, 1985), lo que afecta directamente a la fauna marina. Así, en enero de 2014, en el Pacífico peruano, más de 400 delfines se acercaron a morir a las playas (Foley, 03 de febrero de 2014); del mismo modo, según el portal de noticias ABC, en Nueva Zelanda, en febrero de 2017, más de 400 ballenas murieron (Farewell spit more than 400 whales stranded on New Zealand beach, majority reported dead, 09 de febrero de 2017).

Asimismo, "El Niño", una corriente oceánica fría y de baja salinidad que corre a lo largo de Ecuador, Perú y Chile, ha estado calentándose y alterando el clima en todos los países de la cuenca del Pacífico, y mientras que el fenómeno de "La Niña", se encuentra produciendo sequía en Sudamérica. En los últimos 20 años, el ciclo de estos fenómenos ha ocurrido con mayor frecuencia y violencia. Así, en marzo de 2017, la temperatura de la superficie del mar en la costa norte de Perú pasó de $6{ }^{\circ} \mathrm{C}$ a $27{ }^{\circ} \mathrm{C}$, lo que provocó lluvias intensas que terminaron en grandes inundaciones y destrucción masiva en cuatro departamentos del Norte (Climate Prediction Centre/NCEP, 2017). Para empeorar la 
situación, el deshielo del Ártico está desequilibrando las jet stream (corrientes de aire polar), por lo que las lluvias son bastante menos espaciadas que antes, se precipitan por varios días en el mismo lugar y causan grandes inundaciones. Asimismo, algunos científicos argumentan que la corriente de aire del hemisferio norte cruzó el ecuador hacia el hemisferio sur, lo que amenaza el suministro mundial de alimentos $y$ el fin del invierno.

De acuerdo con Mooney (15 de febrero de 2017), "El mayor volumen de oxígeno se perdió en el océano más grande, el Pacífico, pero como porcentaje, el declive fue más agudo en el océano Ártico, una región que enfrenta el cambio climático más severo de la Tierra" (párr. 2). Sin embargo, en nombre del progreso, "una locura esquizofrénica" impulsada por una forma de productivismo, está tratando de "salvar" el Ártico con los recursos de la geoingeniería. Así, a medida que el hielo del Ártico se derrite, un grupo de investigadores de la Universidad de Arizona planea volver a congelarlo utilizando alrededor de 10 millones de bombas de agua impulsadas por el viento instaladas en la región (Martins, 19 de febrero de 2017). Mientras tanto, la Estación de Investigación del Alto Ártico canadiense (CHARs por sus siglas en inglés), una organización federal, está haciendo un inventario de los recursos contenidos en el Ártico para proporcionar permisos para proyectos mineros y petroleros, con el fin de legalizar esta forma de daño.

En el polo opuesto, en la Antártida, ocurre algo semejante, pues sus glaciares están siendo afectados: las plataformas de hielo en Larcen A, B y C colapsaron en 1995, 2002 y julio de 2017, respectivamente; $y$ "cuando una plataforma de hielo colapsa, los glaciares detrás de ella pueden acelerar su deshielo" (Patel, 07 de febrero de 2017). Estos hechos pueden transformar el paisaje de la península Antártica y promover el colapso de otras plataformas de hielo vulnerables. Las altas temperaturas del Ártico y la Antártida están aumentando a un ritmo dos veces superior al promedio mundial; algo crítico además porque su existencia es fundamental para la estabilidad climática por el efecto albedo, por el que la nieve refleja de regreso los rayos del Sol. Todos estos fenómenos, de modo concomitante, elevan el nivel del mar en directo perjuicio de las comunidades insulares y las áreas costeras bajas.

En resumen, las emisiones totales de carbono en diciembre de 2017 superaron las 406.82 partículas por millón (ppm) en la concentración global de carbono (el máximo debe ser de 300 ppm) (National Oceanic and Atmospheric Administration, s.f. b) y el metano $\mathrm{CH} 4$ total (un gas de efecto invernadero liberado como resultado del deshielo) fue de 1,865 partículas por billón (ppb), cuando el máximo debería ser de 800 (National Oceanic and Atmospheric Administration, s.f. a). Por lo demás, la última evidencia científica afirma que se acerca una catástrofe climática: si continúan aumentando las emisiones de combustibles fósiles, se puede alcanzar un incremento de 2 ${ }^{\circ} \mathrm{C}$ en la temperatura media global, lo que representa un peligroso e irreversible umbral de calentamiento.

En este artículo, el objetivo es examinar las causas del cambio climático y sus severos efectos en los ecosistemas de la Tierra. Se explora a partir de la perspectiva ecofeminista materialista $y$ la perspectiva ecosocialista sobre las diversas formas de explotación de los cuerpos (tanto de los humanos como de los ecosistemas). De esta manera, se concentra en el calentamiento global como consecuencia de la abundante y desproporcionada liberación de dióxido de carbono $\left(\mathrm{CO}_{2}\right)$ en la atmósfera emitido por el funcionamiento de la economía $y$ la militarizacion desarrolladas por la sociedad tecnológica de la que se forma parte. En esa dirección, primero se analiza la crisis reproductiva debido a que la producción y el consumo capitalista de bienes básicos están atacando las capacidades humanas y de la naturaleza hasta el punto de distorsionar los cuerpos orgánicos. Segundo, se investiga las crisis ecológicas generadas por la producción de bienes básicos (excedente) y su consumo (residuos). Tercero, se explora el complejo militar-industrial y sus ramificaciones biofísicas. Cuarto, se presentan dos perspectivas para salir del caos climático. Finalmente, se concluye con la demanda de una solución que incluya a las mujeres, a la 
población campesina, a los pueblos indígenas y a los países llamados del Tercer Mundo, para organizar respuestas políticas colectivas locales e internacionales.

ECOFEMINISMO Y LA CRISIS DE LA REPRODUCCIÓN: ATACANDO LAS CAPACIDADES DE LAS PERSONAS Y LOS ECOSISTEMAS PARA FUNCIONAR COMO ORGANISMOS

Las teorías ecofeministas han contribuido, en particular, al análisis de la dominación masculina de las mujeres y la naturaleza; sin embargo, la reproducción se convirtió en una categoría política en la década de 1970 durante el surgimiento del Movimiento de Liberación de las Mujeres. Maria Dalla Costa y Selma James (1975) argumentan que el "trabajo doméstico" es una condición previa para la producción capitalista, ya que reproduce la fuerza de trabajo $y$ permite el mismo proceso de acumulación de capital. En este contexto, el trabajo reproductivo -invisible como trabajo, impago y realizado en forma aislada - ha socavado la integridad física de las mujeres, por lo que el programa político de estas feministas exigía una remuneración estatal para las tareas domésticas.

Por su parte, las ecofeministas ampliaron el alcance de la teoría feminista para incluir los cuerpos feminizados. Ellas demostraron que ha sido posible mantener la ilusión de que el crecimiento económico es un proceso positivo $y$ benigno porque los costos han sido soportados principalmente por los trabajadores devaluados: las mujeres, los campesinos, los pueblos indígenas, las colonias y la naturaleza. Maria Mies, Veronika BennholdtThomsen y Claudia von Werlhof (1988) describen la labor de las mujeres como el arquetipo del trabajo global no asalariado. También señalan cómo la población trabajadora del Tercer Mundo está involucrada en formas de producción no asalariada, es decir, basadas en el concepto de trabajo doméstico (no pagado o no asalariado). Las autoras describen a los hombres y a las mujeres del Tercer Mundo como las "amas de casa del mundo", de manera que la relación entre marido $y$ mujer se replica en la relación que establecen el Primer y el Tercer Mundo. De esta manera, entienden el trabajo de las mujeres del Primer y Tercer Mundo en los términos del patriarcado y el imperialismo.

Silvia Federici (2004), a su vez, muestra una larga línea histórica desde el movimiento de los comunes y los juicios de brujas hasta la prostitución moderna y la monetización de la naturaleza. Ella teoriza acerca del sometimiento sistemático y la apropiación de los cuerpos y trabajos de las mujeres y la naturaleza; demuestra cómo las relaciones sociales capitalistas son habilitadas por los arreglos patriarcales que separan la producción de la reproducción, el uso de los salarios para comandar el trabajo de los no asalariados y la devaluación del estatus de las mujeres. En esa dirección, la criminalización de los cuerpos de las mujeres se transfirió a las colonias y se generalizó a todos los cuerpos "no blancos". En contraposición, Federici revaloriza el trabajo de reproducción y reconecta la relación con la naturaleza, con los demás y con los cuerpos para recuperar la sensación de plenitud en la vida.

Carolyn Merchant (1990) en The death of nature ofrece una interpretación de la dominación masculina en la ciencia occidental $y$ el progreso industrial; $y$ al examinar la revolución científica propone que el surgimiento de la ciencia, la tecnología y la economía modernas fueron las fuerzas impulsoras de la opresión de las mujeres y la naturaleza. También argumenta que el control masculino de la reproducción, durante la caza de brujas, resultó en el triunfo del mecanismo sobre el organismo y en la relegación del trabajo doméstico a una esfera devaluada. Así también, Vandana Shiva e Ingunn Moser (1995), en Biopolitics demuestran que durante los últimos 70 años, la lógica del mercado ha tomado el control de la economía de subsistencia a través de la ciencia y la tecnología en perjuicio de la capacidad de las personas para funcionar ecológicamente, es decir, como organismos integrados a sus ecosistemas. Las autoras destacan además el hecho de que el paradigma mecanicista de la ingeniería genética ha reducido todo el comportamiento de los organismos biológicos, incluidos los humanos, al ámbito de los genes. Como resultado, la ingeniería genética intercambia genes entre diferentes especies 
$y$ diseña nuevo material genético que no ha existido antes en la naturaleza, lo cual altera la vida sobre la Tierra, pues estas manipulaciones entrañan consecuencias ecológicas negativas debido a que su desarrollo, llevado a cabo en laboratorios, tiene una aplicación y validez limitadas en los sistemas abiertos.

Al discutir los efectos de la ingeniería genética en la humanidad, Stephanie Seneff (2016) afirma que los productos químicos utilizados por Monsanto, como Roundup, están relacionados con nuevas epidemias que están destruyendo los cuerpos de las personas. Ella muestra así evidencia de que la epidemia de autismo contemporánea se debe a la exposición de los cuerpos a tóxicos ambientales, en particular, al glifosato.

... la incidencia del autismo ha aumentado de forma alarmante en las últimas dos décadas en los Estados Unidos, exactamente en sintonía con el uso creciente del Roundup como herbicida en los cultivos de maíz y soja. Mientras que la tasa de autismo se estimó en 1 en 10,000 en 1970, el número más reciente de los Centros para el Control y la Prevención de Enfermedades [CDC por sus siglas en inglés] fue de 1 en 68 para los niños/as de 12 años en 2014. Este número refleja el grupo de edad que nació en 2002; seguramente el número sería mucho mayor para los niños nacidos hoy (Seneff, 2016, p. 77).

La autora concluye:

el glifosato utilizado en el maíz y la soja elimina el manganeso ... [y] otros minerales, incluyendo hierro, zinc, cobalto $y$ magnesio, lo que los hace no disponibles para las plantas, $y$ por lo tanto las plantas tienen deficiencia de estos minerales. Las personas que comen las plantas también se vuelven deficientes ... las deficiencias minerales son la fuente clave de muchas de las dolencias que enfrentan los humanos en el mundo moderno (2016, p. 81).
Teniendo en consideración los resultados de la ingeniería genética aplicada a la naturaleza, el libro The "greening" of Costa Rica. Women, peasants, indigenous people and the remaking of nature (Isla, 2015) informa que los árboles transgénicos plantados (teca, melina y eucalipto) en Costa Rica, con el fin de vender créditos de carbono, han destruido el ecosistema forestal, en particular, afectando su capacidad para regenerar bosques secundarios, que conservan la biodiversidad y regulan la hidrología. Al mismo tiempo, en el marco del crédito de carbono, los bosques son "capital natural" y las comunidades que solían vivir en ellos son declaradas su enemigo. El Gobierno costarricense, a través de su Ministerio de Ambiente y Energía, evalúa la capacidad de los empresarios agrícolas a gran escala en asociación con el capital internacional para vender créditos de carbono. Como resultado, estos árboles son vulnerables al fuego, plagas $y$ enfermedades, $y$ las empresas pueden cortar los árboles después de 15 años de crecimiento $y$ transformarlos en madera para pisos o papel, cajas para exportación de frutas o muebles.

El monocultivo de especies de árboles se ha convertido de este modo en una bomba de tiempo para la biodiversidad en Costa Rica. Por ejemplo, las plantaciones de teca han ocasionado la erosión de las llanuras, ya que su sistema de raíces crece profundamente en el suelo, pero en la selva, los sistemas de absorción de nutrientes y agua están en la superficie, pues en general estos se concentran a una profundidad de entre 70 y 100 centímetros. En consecuencia, los árboles de teca están rodeados de tierra en copos; de forma que cuando llueve, las hojas de gran tamaño acumulan grandes cantidades de agua que luego vierten violentamente en un suelo ablandado, destruyéndolo. Además, la amplia difusión de las raíces y la sombra producida por las hojas obstruyen el crecimiento vegetativo en la capa inferior del bosque, lo que podría evitar el daño del suelo a causa de las cascadas violentas. Bajo la teca, la comida para bacterias, virus $y$ personas ha desaparecido. Estos árboles no pueden reemplazar a los naturales porque estos están conectados directamente entre sí a través de la multitud 
de criaturas que se relacionan con ellos como alimento, refugio o lugar de anidamiento a través de su acceso compartido al agua, aire $y$ luz solar, y por medio de un sistema subterráneo de hongos que vincula a todos los árboles como un superorganismo. En consecuencia, al plantar bosques homogéneos genéticamente modificados, los ecosistemas de la selva tropical costarricense se están convirtiendo en monocultivos estériles.

Para concluir, las ecofeministas han señalado que la economía política de la ciencia y la tecnología, aplicada a la producción y al consumo de bienes básicos, están distorsionando los cuerpos humanos y naturales en el planeta. La siguiente sección discute la economía política de la crisis ecológica en relación con la Física, la Química y la Biología. Al respecto, los ecosocialistas argumentan que, en términos bioeconómicos, cada proceso económico debe verse desde el punto de vista de los principios de la termodinámica, pues aseguran que estos principios se aplican tanto a los sistemas naturales como a los sociales. La actividad económica no escapa así a las leyes de la Física, e incluso, los seres vivos están sujetos a la entropía.

\section{ECOSOCIALISMO Y LAS CRISIS ECOLÓGICAS GENERADAS POR LA PRODUCCIÓN DE BIENES BÁSICOS (EXCEDENTE) Y SU CONSUMO (RESIDUOS)}

El análisis empírico y los datos históricos confirman la existencia de una estrecha conexión entre las cantidades de energía fósil consumida, el estado de la actividad asalariada y el funcionamiento de la economía en general. El consumo creciente de materiales primarios afecta al clima, principalmente debido a las grandes cantidades de energía involucradas en su extracción, uso, transporte y residuo (basura), todas actividades contaminantes. Jean-Paul Deleage (1994) asegura que el planeta está siendo destruido por la producción y consumo iniciados hace mucho tiempo, proceso acelerado por el sistema capitalista y en particular por la globalización del capital y la intervención de su complejo militar-industrial. Él argumenta que el capitalismo siempre ha tratado a la naturaleza como ilimitada, por lo tanto, aceleró la irreversibilidad entrópica en el planeta; tambien sostiene que en "términos bioeconómicos, la extracción de materias primas y su eliminación en forma de residuos constituyen la primera y la última fase de toda la actividad económica" (1994, p. 39). En consecuencia, los problemas sociales y ecológicos surgen al comienzo de la producción, en la primera fase de toda la actividad económica, cuando las materias primas se extraen $y$ se consumen; $y$ concluyen cuando se descargan en el medio ambiente en forma de residuos, la última fase de toda actividad económica.

Los ecosocialistas sostienen que históricamente el calentamiento global empieza en Inglaterra, cuando el acceso de los británicos a las materias primas de sus colonias y el sistema de fábricas textiles basado en el agua, les permitieron producir en cantidades nunca antes vistas. Sin embargo, todo cambió cuando el trabajador artesanal fue reemplazado por un motor como el eje central de la fábrica, con una constante y creciente demanda de energía. El carbón proveyó esa energía - un combustible con un alto contenido de carbono como motor primario mecánico-y fomentó la revolución industrial. Desde entonces, la liberación de metano de los combustibles fósiles ha aumentado la entropía planetaria a niveles amenazantes.

Andreas Malm (2016), en Fossil capital, asegura que "[con el] carbón colocado justo bajo el fuego de la acumulación de capital ... una espiral de creciente combustión de combustibles fósiles fue, por primera vez, integrado en el crecimiento en espiral de la producción de mercancías" (p. 292). El autor llama "capital fósil" a la asociación de la quema de combustibles fósiles (la principal causa del cambio climático inducido por el hombre) con el desarrollo capitalista de la producción industrial. El incremento del uso de combustible fósil para la producción de bienes básicos ha conducido así a una sucesión de interrupciones de los antiguos equilibrios ecológicos locales y regionales. Por ejemplo, en 1952, Londres se vio afectada por la severa contaminación del aire derivada del uso de carbón (Davis, Bell y Fletcher, 2002). 
Elmer Altvater (1994) encontró importantes fuentes de contradicción en el funcionamiento del capitalismo luego de identificar su intento de codificar y controlar el tiempo $y$ el espacio con el objetivo de acelerar el proceso de acumulación de capital, definido como modalidad económica. Sin embargo, el proceso natural del tiempo y el espacio, ajeno a esta manipulación, es descrito como modalidad ecológica. Altvater sostiene que estas dos modalidades, la económica y la ecológica, entran en conflicto en el marco de una realidad territorial-social, ya que el tiempo biológico y la reproducción son más lentos que el tiempo económico o la producción de mercancías. Como el capitalismo es un sistema que solo entiende el valor en términos de capital monetario, el impulso perpetuo hacia la acumulación, "t" (tiempo económico), a corto plazo está en conflicto directo con los límites ecológicos de "T" (tiempo histórico), que permiten y proporcionan la vida en este planeta.

Al aplicar los principios de la entropía al uso de la energía para la producción y el consumo, donde ninguna transformación de energía o materia es perfectamente eficiente, Altvater concluye que el reciclaje es termodinámicamente imposible, por lo que la energía y las materias primas se usan una sola vez. Como resultado de este conflicto ecológico-económico, la tasa de extinción se ha acelerado desde la expansión del desarrollo capitalista industrial $y$ ha creado la sexta extinción masiva de la biodiversidad en curso, que elimina al menos 150 especies por día. Gerardo Ceballos, Paul Ehrlich y Rodolfo Dirzo (2017) indican así "que más allá de las extinciones globales de especies, la Tierra está experimentando un gran episodio de disminución y extirpación de la biodiversidad, que tendrá consecuencias negativas en cascada sobre el funcionamiento del ecosistema y los servicios vitales para sostener la civilización" (p.7). Por ejemplo, hondureños y guatemaltecos están a las puertas de EE. UU. porque su hábitat ha sido reducido por la minería, principalmente canadiense (Grahame, 2018).

\section{EL COMPLEJO MILITAR-INDUSTRIAL Y SUS RAMIFICACIONES BIOFÍSICAS}

El cambio climático no se hubiera agudizado tanto como lo hizo en los últimos 70 años sin el dominio del complejo militar-industrial, empeñado en emprender una guerra de contrainsurgencia (Pursell, 1972) que aterroriza a las poblaciones (Castaneda, 1993), reprime la disidencia interna (Burns, 2018) y destruye la capa atmosférica (Bertell, 2001). En clara muestra de desprecio por el valor de la vida, la expansión mundial de la política del capital global, neocolonial y neoliberal se organizó a través del complejo militar-industrial, definido por Carroll Pursell (1972) como:

... una coalición informal y cambiante de grupos con intereses psicológicos, morales $y$ materiales adquiridos en el continuo desarrollo y mantenimiento de altos niveles de armamento, en la preservación de los mercados coloniales $y$ en el sector estratégico-militar de los asuntos internacionales. Estos grupos incluyen ...congresistas, líderes laborales, ejecutivos de corporaciones, portavoces de la Iglesia, profesores universitarios y soldados profesionales... En el centro del complejo... está la relación única entre el Pentágono como comprador y las industrias de guerra como vendedores (p. IX).

Pursell (1972) afirma que después de la Segunda Guerra Mundial, la decisión básica fue adaptar el programa de guerra a la estructura económica existente para apoyar al comercio: "Un mundo pacífico y seguro para el comercio estaría en adelante garantizado por un establecimiento militar permanente respaldado por una economía de guerra preparada para ofrecer un apoyo masivo a las intervenciones extranjeras" (p. 7).

Desde la década de 1960, el complejo militar-industrial ha sido protagonista del nuevo imperialismo, ya que mejora la capacidad bélica de los EE. UU. e incrementa su dominio sobre las personas y la naturaleza, lo cual "aumenta la probabilidad de recurrir a 'soluciones' basadas en el poder militar” (Melman, 1972, p. 290). 
Actualmente, el capital global, liderado también por los EE. UU., a través de la guerra se ha apoderado del petróleo de Irak y Libia en el Medio Oriente, así como de minerales en Afganistán, $y$ actualmente, se encuentra Venezuela en su mira (Graham-Harrison y Parkin, 25 de febrero de 2019).

Las ramificaciones biofísicas de la alienación militar capitalista son discutidas por la ecofeminista Rosalie Bertell (1985) en su libro No Immediate Danger?: Prognosis for a Radioactive Earth, donde sostiene que los militares de EE. UU. se han estado preparando para una guerra climática mediante la "armamentización" de todos los sistemas que sostienen la vida. Esta autora - crítica de la guerra $y$ la industria nuclear del complejo militarindustrial, que comienza en el laboratorio de investigación pero termina en la atmósferaafirma que, durante la Guerra Fría, el Reino Unido, los EE. UU., Francia y los soviéticos exploraron la atmósfera con intenciones bélicas. Bertell proporciona evidencia de las pruebas atmosféricas nucleares de los EE. UU. llevadas a cabo en el océano Pacífico desde 1946. De esta forma, durante diez años, activaron más de 86 bombas nucleares y desde 1983 también han lanzado misiles en las Islas Marshall de Micronesia (también en el Pacífico). La autora detalla además que entre 1966 y 1974, el Centre d'Expérimentation du Pacifique de Francia realizó 7 pruebas nucleares en el océano Pacífico. Como resultado de la explosión de estas bombas, se argumenta que el campo magnético de la Tierra - los cinturones de Van Allen, que protegen al planeta del viento solar destructivo cargado de partículas - ha sido dañado. Estas perturbaciones electromagnéticas también pueden contribuir a la contaminación actual y explicar la reducción de oxígeno en el Pacífico, en el cual se encuentran mamíferos muertos en su orillas (véase la introducción).

Bertell relata también que en 1974, Perú denunció las consecuencias de la radiación en las Naciones Unidas y rompió relaciones diplomáticas con Francia porque las pruebas envenenaron a sus pescadores y ciudadanos. Desde entonces, El Niño o Corriente de Humboldt ha estado calentando y alterando el clima en todos los países de la costa del Pacífico, además de afectar a Australia, Nueva Zelanda y Fiji (Bertell, 1985).

Tiempo después, en el año 2001, Bertell sostuvo en su libro Planet Earth. The latest weapon of war que "el objetivo de dominación de los militares de EE. UU. se extiende al calentamiento atmosférico en la ionosfera, y está experimentando con el clima que distorsiona los cuerpos orgánicos" (p. 125). Así, después de la Segunda Guerra Mundial, los militares investigaron y manipularon la atmósfera con productos químicos para generar lluvias, huracanes, tornados y monzones. Lowell Ponte (1976), en The cooling: Has the next ice age already begun?, proporciona evidencia de que la modificación del clima comenzó durante la Guerra de Vietnam, cuando los EE. UU. investigaron formas de usar rayos artificiales como arma y maneras de provocar huracanes, con los proyectos Skyfire y Stormfury, respectivamente. También investigaron la posibilidad de destruir la capa de ozono sobre Vietnam del Norte con láseres o productos químicos con el fin de causar daño a cultivos y humanos. Bertell agrega que "se rociaron millones de galones [del Agente Naranja] en más del 10 por ciento de Vietnam, reduciendo las densas selvas y los bosques de manglares a terrenos baldíos. Muchos niños en las áreas contaminadas nacieron con dificultades de aprendizaje o con deficiencias graves" (2001, p. 158).

Además, Bertell descubrió una red militar de calentadores ionosféricos que dispara ondas electromagnéticas que pueden dañar seriamente al ecosistema. De esta forma, el Programa de Investigación Auroral Activa de Alta Frecuencia (HAARP por sus siglas en inglés) modifica la ionosfera al servicio de una agenda de dominación militar, cuyo objetivo principal es la manipulación del electrojet aural ${ }^{1}$

1 El HAARP, programa de la Fuerza Aérea norteamericana, tiene su base en Gakona, Alaska, donde ha instalado 180 antenas que en conjunto emiten 1 $\mathrm{GW}=1.000 .000 .000 \mathrm{~W}$, es decir, un billón de ondas de radio de alta frecuencia, las cuales penetran en la atmósfera inferior e interactúan con la corriente de los electrojets aureales (véase https://judith79. wordpress.com/tag/electrojet-aural/). 
como un arma capaz de redirigir cantidades significativas de energía electromagnética hacia el enemigo. Como un uso adicional, esta herramienta es útil para la generación de ondas de baja frecuencia como las empleadas en las comunicaciones por radio, Internet, teléfonos celulares, televisión satelital y submarinos. Sin embargo, aunque los transmisores masivos del HAARP producen incisiones en la ionosfera que son reparadas naturalmente por el Sol, Bertell sostiene que estos "cortes" hechos por el hombre "pueden desestabilizar un sistema que ha mantenido su propio ciclo durante millones de años" (2001, p. 123).

Asimismo, la autora refiere que la Declaración Federal de Impacto Ambiental del HAARP, presentada por la Fuerza Aérea, informa que sus transmisiones "pueden elevar la temperatura corporal interna de las personas cercanas, encender llamas en los baúles de los automóviles, detonar municiones aéreas usadas en fusibles electrónicos, destruir las comunicaciones de aeronaves, navegación y sistemas de control de vuelo" (2001, p. 126). Asimismo, las ondas de baja frecuencia del HAARP, que son reflejadas de regreso a la Tierra por la ionosfera, crean vibraciones a grandes distancias subterráneas que podrían inducir efectos como los terremotos, así como generar efectos climáticos directos. Alrededor del HAARP hay otras redes militares, como superDARns, que monitorea los calentadores ionosféricos en la atmósfera inferior, y GWEN (Ground Wave Emergency Network), que genera tormentas, así como un sistema de guía de armas y drones.

Además, Bertell descubrió que, desde la década de 1990, los EE. UU. y sus aliados contaminaron deliberadamente el medio ambiente como estrategia de guerra en Kosovo e Irak para probar sus armas militares más recientes, basadas en la artillería de uranio empobrecido (DU). Por su lado, la Organización del Tratado del Atlántico Norte (OTAN) usó también bombas de Du y misiles en la guerra de Kosovo. Cuando se dispara, el Du se quema a más de 3000 grados centígrados y se convierte en un aerosol de uranio que contiene partículas radioactivas microscópicas que contaminan toda la vida (tierra, aire y agua), y continúan haciendo daño mucho tiempo después de ser usados. Así, la dependencia de las potencias respecto del petróleo del Golfo Pérsico hizo que la OTAN lanzara 88000 toneladas de explosivos (7 veces la fuerza de la bomba de Hiroshima) sobre Irak. A su vez, los EE. UU. utilizaron "armas de calor" que emplean energía solar para quemar el país. Al mismo tiempo, los ataques militares combinados de las fuerzas estadounidenses e iraquíes destruyeron oleoductos, pozos de petróleo, refinerías y tanques de almacenamiento. Bertell informó que como consecuencia se formaron enormes nubes de humo tóxico y se liberó al menos 40 toneladas de polvo radioactivo en los campos de batalla de Kuwait e Irak. Este impacto en la atmósfera produjo fuertes tormentas, tifones, inundaciones y lluvia ácida en toda esa parte del mundo. El pronóstico de Bertell es que los militares se están preparando para una guerra climática.

El Grupo de Acción sobre Erosión, Tecnología y Concentración (ETC Group) ha confirmado la militarización a escala global y la "armamentización" de todos los medios de vida mediante la manipulación del clima. Sin embargo, a pesar de que ninguna de las condiciones aquí descritas que tienen efecto en el cambio climático puede superarse con la tecnología, la economía productivista de los colonizadores propone tomar el control del termostato global a través de la geoingeniería "civil". Según el ETC Group (2010), esta geoingeniería responde al enfoque tecnológico diseñado para intervenir a gran escala en los océanos, suelos o atmósfera de la Tierra para reducir el $\mathrm{CO}_{2}$. Esta intervención se concreta en una amplia gama de acciones que ya son una realidad - pero sin que el público sea informado-, que incluyen: liberar partículas de sulfato en la estratosfera para reflejar de vuelta los rayos del Sol, verter partículas de hierro en los océanos para que el plancton absorba el $\mathrm{CO}_{2}$, disparar yoduro de plata a las nubes para producir lluvia, así como cultivar plantas y árboles genéticamente modificados para que su follaje refleje mejor la luz solar (ETC Group, 2010). Además:

... el 15 de abril de 2017, el programa de geoingeniería más grande hasta la fecha dio inicio oficialmente a sus actividades 
en la Universidad de Harvard, y ha recaudado más de US\$ 7 millones hasta el momento, con el objetivo de usar US\$ 20 millones durante siete años. Este programa incluirá una fase de prueba de campo durante la que los científicos pulverizarán partículas diminutas y altamente reflectantes a altitudes de más de siete millas sobre el desierto de Arizona para probar la eficacia de uno de los métodos de geoingeniería más conocidos, llamado gestión de la radiación solar (Freedman, 19 de abril de 2017, párr. 5).

Los actores que lideran el avance de la geoingeniería son los militares, las universidades, la Royal Society del Reino Unido y la Academia Nacional de Ciencias de EE. Uu., junto con Canadá, Alemania y Rusia. No obstante, la geoingeniería supone altos riesgos ambientales que pueden llevar más rápido a la extinción del planeta.

En la siguiente parte, se presentan algunas propuestas para solucionar el caos climático desde aquellas voces que han sido silenciadas.

\section{LA SALIDA DEL CAOS CLIMÁTICO DESDE DOS PERSPECTIVAS: ECOCÉNTRICA Y DE SUBSISTENCIA}

En las tres perspectivas presentadas, lo primero es salir del marco de producción de mercancías para dar una solución al caos climático. Joel Kovel (2007), autor de The enemy of nature, sugiere una reestructuración de la sociedad basada en un modo de producción ecocéntrico que incluya una nueva teoría del valor, que él llama "valores intrínsecos". Kovel propone liberar el valor de uso del valor de cambio y abrir la apropiación del valor intrínseco para construir la integridad del ecosistema, así como cambiar las relaciones de propiedad para que la Tierra, vista como la fuente de todos los valores de uso y todos los ecosistemas, sea apropiada por los "productores asociados". Él sostiene también que la prefiguración (una forma de organizar las sociedades) "es intrínseca a la producción ecológica, haciendo que la producción de subsistencia (o aprovisionamiento) del ecofeminismo sea la previsión de un momento utópico" (Kovel, 2007, p. 218). Kovel se inclina además por actuar en el marco de la cosmovisión indígena y el aprovisionamiento del ecofeminismo para dirigir la salida de las crisis ecológicas y sociales.

La postura ecofeminista considera que las economías locales de subsistencia son un modelo alternativo de justicia social, económica, de género y ecológica. Por ejemplo, Maria Mies y Veronika Bennholdt-Thomsen (1999) proponen "la perspectiva de subsistencia", que se basa en la producción que incluye todo el trabajo invertido en la creación y mantenimiento de la vida inmediata, sin ningún otro propósito. Se trata de una forma de resistencia a los efectos del patriarcado, el capitalismo global y el colonialismo. Los productores de subsistencia en todo el mundo - la mayoría de los cuales son mujeres o feminizadas - deben ser vistos como expertos que pueden liderar el camino hacia la recuperación de modos de vida autónomos, comenzando desde la autonomía territorial y corporal, la seguridad alimentaria en pequeñas granjas y la eficiencia energética.

Por su parte, Ariel Salleh (2004) desarrolló el concepto de trabajadores metaindustriales, entendidos como los miembros no remunerados o mal pagados de los hogares, los campesinos y los pueblos indígenas, con una capacidad única de aprovisionamiento, lo que significa que su trabajo satisface sus necesidades ecológicas y culturales. El análisis de Salleh (2004) se llama Materialismo Personificado. $\mathrm{Su}$ argumento es que los cuerpos feminizados, como el de las mujeres, son definidos como un "recurso natural" para reproducir la fuerza de trabajo; $y$ los cuerpos de los campesinos, comunidades indigenas y países colonizados han sido utilizados como recursos por su sexualidad, su trabajo esclavo, su ADN, etc. Estos miembros de la sociedad y sus conocimientos han sido marginados, censurados y reprimidos por las vanidades de la modernidad. Asimismo, el autor sostiene que la economía sociocultural (de subsistencia) es una esfera ecosuficiente de trabajo regenerativo y valor de uso, $y$ es más importante para la economía que la esfera de producción para el intercambio. 


\section{CONCLUSIÓN}

Las crisis descritas en este artículo son fruto de la racionalidad económica del capital global-patriarcal-colonialista que ha concentrado la riqueza, el poder político y militar, mientras ha ignorado la finitud de los recursos y el declive del planeta Tierra, así como, los cuerpos feminizados, asalariados y no asalariados, oprimidos y explotados.

La acumulación de capital, en su afán de expandir sus operaciones para abarcar todo el planeta, ha interrumpido el proceso biogeoquímico-físico de la Tierra, expresado de forma dramática en el cambio climático. La comunidad científica sostiene que se ha llegado al punto de no retorno al equilibrio del ecosistema. La salida a esta crisis creada por el poder económico incluye a todas aquellas voces que han sido silenciadas, como las de las mujeres, los campesinos y los pueblos indígenas de las excolonias. Actualmente, la última respuesta a este caos es una revolución social y ecológica de amplia base, en la cual todos y todas se movilicen para proteger el futuro de la humanidad en la Tierra.

\section{REFERENCIAS}

Alberta Government. (10 de junio de 2016). Final update 39: 2016 Wood Buffalo Wildfires. https://www.alberta.ca/release. cfm?xID=41701E7ECBE35-AD48-57931642C499FF0DE4CF

Altvater, E. (1994). Ecological and economic modalities of time and space. En M. O'Connor. (Ed.), Is capitalism sustainable? Political economy and the politics of Ecology (pp. 76-90). The Guilford Press.

Bertell, R. (1985). No immediate danger: Prognosis for a Radioactive Earth? The Women's Educational Press.

Bertell, R. (2001). Planet Earth. The latest weapon of war. Black Rose Books.

Burns, S. (2018). Revolts and the military in the Arab Spring: Popular uprising and the politics of repressions. I. B. Tauris \& Co. Ltda.

Castaneda, J. (1993). The utopia unarmed. The Latin American left after the Cold War. Vintage Books.
Ceballos, G., Ehrlich, P. y Dirzo, R. (2017). Biological annihilation via the ongoing sixth mass extinction signaled by vertebrate population losses and declines. Proceedings of the National Academy of Sciences of the United States of America, 114(30). https://www.pnas.org/content/114/30/E6089

Climate Prediction Centre/NCEP (2017). ENSO Alert System Status: El Nino Watch. ENSO: Recent Evolution, Current Status and Predictions.

Dalla, M. y James, S. (1975). The power of women and the subversion of the community. Falling Wall Press.

Davis, D. L., Bell, M. L. y Fletcher, T. (2002). A look back at the London smog of 1952 and the half century since. Environmental Health Perspectives, 110 (12), A734-A735. doi: 10.1289/ ehp.110-a734

Davis, J. (2017). Low oxygen levels in the ocean could led to more wildfires in the future. Iflscience! http://www.iflscience.com/ environment/low-oxygen-levels-in-theocean-could-lead-to-more-wildfires-inthe-future/

Deleage, J. P. (1994). Eco-marxist critique of political economy. En M.O'Connor. (Ed.), Is capitalism sustainable? Political economy and the politics of ecology (pp. 37-52). The Guilford Press.

ETC Group. (2010). Geopiracy: The case against geoengineering. http://www.etcgroup. org/sites/www.etcgroup.org/files/publication/pdf_file/ETC_geopiracy_4web.pdf

Farewell spit more than 400 whales stranded on New Zealand beach, majority reported dead. (09 de febrero de 2017). ABC News. http://www.abc.net.au/news/2017-02-10/ more-than-400-whales-strand-on-newzealand-beach-most-die/8258236

Federici, S. (2004). Caliban and the witch: women, the body and primitive accumulation. Automedia.

Foley, J. (03 de febrero de 2014). More than 400 dolphins washed ashore dead in Peru in January. Nature World News. http:// www.natureworldnews.com/articles 
/5861/20140203/more-400-dolphinswashed-ashore-dead-peru-january.htm

Freedman, A. (19 de abril de 2017). We're about to test out hacking the Earth's climate. That should scare or inspire you. Yahoo! News. https://www.yahoo.com/news/apostry-hack-earth-apos-213339241.html

Gobierno de Canadá. (06 de mayo de 2016). Daily Data Report. Environment Canada. http://climate.weather.gc.ca/climate_data/daily_data_e.htmlStationID= $27214 \&$ Month $=12 \&$ Day $=1 \&$ Year $=2016 \& \mathrm{t}$ imeframe $=2 \&$ StartYear $=1840 \&$ EndYear= 2016\&wbdisable

Grahame, R. (2018). Violence, evictions \& impoverishment of global economy are political persecution: The U.S., Canada \& 'international community' helping produce forced migrancy flight from Honduras \& Guatemala. [Mensaje en un blog] Rights Action. https://rightsaction. org/blog/

Graham-Harrison, E. y Parkin, J. (25 de febrero de 2019). Venezuela: Guaidó wants 'all options' open as he meets Pence. The Guardian. https://www.theguardian. com/world/2019/feb/24/venezuela-standoff-juan-guaido-nicolas-maduro

half century since. Environmental Health Perspectives, 110 (12), A734-A735. doi: 10.1289/ehp.110-a734

Intergovernmental Panel on Climate Change [IPCC]. (Octubre, 2018). Special Report. Global Warming of 1.5. The Report of Intergovernmental Panel on Climate Change. https://www.ipcc.ch/sr15/

Isla, A. (2015). The "greening" of Costa Rica: women, peasants, indigenous people and the remaking of nature. The University of Toronto Press.

Kovel, J. (2007). The enemy of nature: the end of capitalism or the end of the world? Fernwood Publishing.

Lowell, P. (1976). The Cooling: Has the Next Ice Age Already Begun? Can We Survive It? Prentice Hall.

Malm, A. (2016). Fossil capital: the rise of steam power and the roots of global warming. Verso.
Martins, D. (19 de febrero de 2017). The plan to refreeze the Artic with millions of wind pumps. Digital Reporter. https://www. theweathernetwork.com/news/articles/ how-to-re-freeze-the-arctic-install-millions-of-wind-pumps/79552

McPerson, G. (31 de mayo de 2018). Methane unveiled: Q\&A on abrupt climate change [video]. YouTube. https://www.youtube. $\mathrm{com} /$ watch? $v=$ iamBlLIE9P0

Melman, S. (1972). Pentagon capitalism. En C. W. Pursell. (Ed.), The military-industrial complex (pp. 286-296). Harper \& Row.

Merchant, C. (1990). The death of nature: women, ecology and the scientific revolution. Harper and Row.

Mies, M. y Bennholdt-Thomsen, V. (1999). The subsistence perspective: beyond the globalized economy. Zed Books.

Mies, M., Bennholdt-Thomsen, V. y Von Werlhof, C. (1988). Women: the last colony. Zed Books.

Mooney, C. (15 de febrero de 2017). Scientists have just detected a major change to the Earth's oceans linked to a warming climate. The Washington Post. https://www.washingtonpost.com/news/ energy-environment/wp/2017/02/15/ its-official-the-oceans-are-losing-oxygen-posing-growing-threats-to-marinelife/?tid=pm_business_pop\&utm_term $=$. f36clfa6cb35

National Aeronautics and Space Administration [NASA] (18 de enero de 2017). NASA, NOAA Data Show 2016 Warmest Year on Record Globally. [comunicado de prensa]. https://www.nasa.gov/press-release/ nasa-noaa-data-show-2016-warmestyear-on-record-globally

National Aeronautics and Space Administration [NASA]. (14 de mayo de 2014). What is climate change? [comunicado de prensa]. https://www.nasa.gov/audience/ forstudents/k-4/stories/nasa-knows/ what-is-climate-change- $k 4$.html

National Oceanic and Atmospheric Administration [NOAA]. (s.f. a). Trends in atmospheric carbon dioxide. Earth System Research Laboratory, Global 
Monitoring Division. https://www.esrl. noaa.gov/gmd/ccgg/trends/

National Oceanic and Atmospheric Administration [NOAA]. (s.f. b). Mauna Loa, Hawaii Observatory. Earth System Research Laboratory, Global Monitoring Division. https://www.esrl.noaa.gov/gmd/obop/mlo/

Patel, J. K. (07 de febrero de 2017). A crack in an Artic ice shelf grew 17 miles in the last two months. The New York Times. https://www.nytimes.com/ interactive/2017/02/07/science/earth/ antarctic-crack.html? smid = twnytimes\&smtyp=cur\&_r $=1$

Pursell, C. W. (1972). The military-industrial complex. Harper \& Row.

Sachs, W. (2010). Environment. En W. Sachs. (Ed.), The development dictionary. A guide to knowledge as power (pp. 24-37). Zed Books.

Salleh, A. (2004). Global alternatives and the meta-industrial class futures beyond globalization. Taylor \& Francis.

Sánchez, K. (11 de julio de 2015). Ola de frío en región Puno deja 21 fallecidos y el deceso de 171 mil alpacas. La República. http://larepublica. pe/impresa/sociedad/14745-ola-de-frioen-region-puno-deja-21-fallecidos-y-eldeceso-de-171-mil-alpacas

Seneff, S. (2016). Autism and gliphosate: connecting the dots. En V. Shiva. (Ed.), Seed sovereingty. Food security. Women in the vanguard of the fight against GMOS and corporate agriculture (pp. 77-103). North Atlantic Books.

Shiva, V. y Moser, I. (Eds.). (1995). Biopolitics. A feminist and ecological reader on biotechnology. Zed Books.

Steffen, W., Crutzen, P. y McNeill, J. (2007). The Anthropocene. Are humans now overwhelming the great forces of nature? Ambio, 36(8), 614-621.

World Commission on Environment and Development [WCED]. (1987). Our common future. Oxford University Press.

Fecha de ingreso: 08/08/2018 Fecha de aprobación: 27/09/2019 